\title{
Clinical Outcomes of Patients Who Perform High-Load Activities after Total Hip Arthroplasty in Snowy Agricultural Areas
}

\author{
Tetsuya Kawano ${ }^{1,2}$, Hiroaki Kijima ${ }^{2,3}$, Toshiaki Aizawa1, Shin Yamada², Natsuo Konishi2, \\ Hitoshi Kubota², Hiroshi Tazawa², Takayuki Tani², Norio Suzuki², Keiji Kamo², \\ Yoshihiko Okudera ${ }^{2}$, Ken Sasaki ${ }^{2}$, Masashi Fujiii ${ }^{2,3}$, Yosuke Iwamoto ${ }^{2,3}$, \\ Itsuki Nagahata2,3, Takanori Miura², Naohisa Miyakoshi' ${ }^{3}$, Yoichi Shimada ${ }^{2,3}$ \\ ${ }^{1}$ Department of Orthopedic Surgery, Kita-Akita Municipal Hospital, Akita, Japan \\ ${ }^{2}$ Akita Hip Research Group, Akita, Japan \\ ${ }^{3}$ Department of Orthopedic Surgery, Akita University Graduate School of Medicine, Akita, Japan \\ Email: *iwtbagdtk@gmail.com
}

How to cite this paper: Kawano, T., Kijima, H., Aizawa, T., Yamada, S., Konishi, N., Kubota, H., Tazawa, H., Tani, T., Suzuki, N., Kamo, K., Okudera, Y., Sasaki, K., Fujii, M., Iwamoto, Y., Nagahata, I., Miura, T., Miyakoshi, N. and Shimada, Y. (2018) Clinical Outcomes of Patients Who Perform High-Load Activities after Total Hip Arthroplasty in Snowy Agricultural Areas. Surgical Science, 9, 248-255.

https://doi.org/10.4236/ss.2018.98030

Received: July 16, 2018

Accepted: August 11, 2018

Published: August 14, 2018

Copyright $\odot 2018$ by authors and Scientific Research Publishing Inc. This work is licensed under the Creative Commons Attribution International License (CC BY 4.0).

http://creativecommons.org/licenses/by/4.0/

\begin{abstract}
Background: In snowy agricultural areas, weeding and snow shoveling are essential activities, and sports activities are common recreational activities. Clarifying the relationships between the degrees of these activities after total hip arthroplasty (THA) and the clinical outcomes may enable us to predict the results of THA for high-load activity patients. Methods: The subjects were 227 post-THA patients who were followed up between 2012 and 2014 . Patient-reported outcomes were investigated by the Japanese Orthopaedic Association Hip Disease Evaluation Questionnaire (JHEQ) and a questionnaire on postoperative activity. In addition, the Japanese Orthopaedic Association Hip Score (JOA score) was used to quantify hip function. Results: Patients who resumed activities had significantly higher JOA and JHEQ scores than patients who had given up their activities. However, $25.8 \%$ of patients had not resumed weeding with a squatting posture. Furthermore, $20 \%$ had not resumed sports activities. The most common reason for giving up these activities was fear of implant failure. Conclusions: If the patients were informed that there is no problem restarting their activities after THA, the patients could resume activities and their clinical outcomes may be improved.
\end{abstract}

\section{Keywords}

Total Hip Arthroplasty, Postoperative Activities, Weeding, Snow Shoveling 


\section{Introduction}

Total hip arthroplasty (THA) is a standard procedure to relieve the pain of hip osteoarthritis, and the postoperative results of THA are stable. Therefore, patient satisfaction in terms of activities of daily living is high [1] [2]. On the other hand, in agricultural areas and regions with heavy snowfalls in Japan, weeding and snow shoveling are essential activities of daily living. These activities remain essential even for patients who have undergone THA, though this work imposes a heavy burden on the hip joint.

Furthermore, an adequate level of physical activity affects both mental and physical human health. Its role has been documented in both preventive healthcare and supporting treatment of the cardiovascular system, obesity, and diabetes mellitus [3] [4]. Due to changing lifestyles and patients' willingness to be involved in sports activities, a return to sports activities is very important.

If many patients in agricultural areas have to give up these activities, and if long periods are needed to resume routine activities, a modified surgical procedure and post-THA rehabilitation may be needed. Therefore, this study aimed to investigate the return rate and the time required to resume high-load daily activities such as weeding, snow shoveling, and sports activities after THA.

In addition, clarifying the relationships between the degrees of these activities after total hip arthroplasty (THA) and the clinical outcomes may enable us to predict the results of THA for high-load activity patients. Thus, the other purpose of this study was to investigate the associations between clinical outcomes and post-THA activities in snowy agricultural areas.

\section{Materials and Methods}

A total of 227 post-THA patients (280 hips) who consulted six hospitals in one Japanese prefecture in March 2012, August 2013, March 2014, and August 2014 were investigated. The sample selected could represent the whole population in snowy agricultural are, because these 6 hospitals are scattered in a wide area. All of the patients cooperated in a questionnaire survey described below. The patients' average age was 69 years (range, 28 - 89 years); 35 were males, and 192 were females. Approval for this study was given by the institutional committee of our group, and informed consent was obtained from each participant. Patient-reported outcomes of postoperative activities were assessed using the Japanese Orthopaedic Association hip disease evaluation questionnaire (JHEQ) [5]. The JHEQ has been developed in Japan as a tool for evaluating patients' quality of life. This questionnaire consists of three subscales: pain; movement; and mental. The Japanese Orthopaedic Association Hip Score (JOA score) was used as a hip functional score. JOA score are objective score evaluated by health professions. Furthermore, an original questionnaire on postoperative activities developed by our group was also administered. The questionnaire included daily activities, such as weeding while sitting in a chair, weeding in a squatting posture, weeding while standing, snow shoveling with a snow scoop, snow shoveling 
with a spade, dealing with roof snowfall, farm work, and rice reaping. Furthermore, it included sports activities such as ground golf, Japanese croquet, curling, underwater walking, fishing, picking edible wild plants, training using a balance ball, and kendo, in addition to sports activities as previously reported by Healy [6]. The patients were asked to choose those activities that they had done at least once after THA. All patients included in the current study had all clinical data available for analysis.

\section{Statistical Analyses}

Statistical analyses were conducted using the Statistical Package for the Biosciences (SPBS V9.67; Akita University Graduate School of Medicine, Akita, Japan) [7]. Comparisons were done by the Mann-Whitney $U$ test and the Chi-squared test. Differences with p values less than 0.05 were considered significant.

\section{Results}

The average JOA score was $77.4 \pm 13.6 / 100$ (range, $24-100$ ), and the average total JHEQ score was $51.9 \pm 17.2 / 84$ (range, 4 - 84). Patients who resumed their high-load daily activities had significantly higher JOA and JHEQ scores than patients who had given up those activities (Figure 1). Furthermore, the JOA and JHEQ scores of patients restarting sports activities after THA were significantly higher than those of patients who did not resume sports activities (Figure 2). The rate of patients who were involved in high-load daily activities increased sharply more than 1 year postoperatively. Furthermore, the rate of patients who performed high-load daily activities in the postoperative periods $>1$ year but $<2$ years and $>3$ years but $<4$ years was significantly higher than in the postoperative period $<1$ year $(\mathrm{P}<0.05)$ (Figure 3 ).

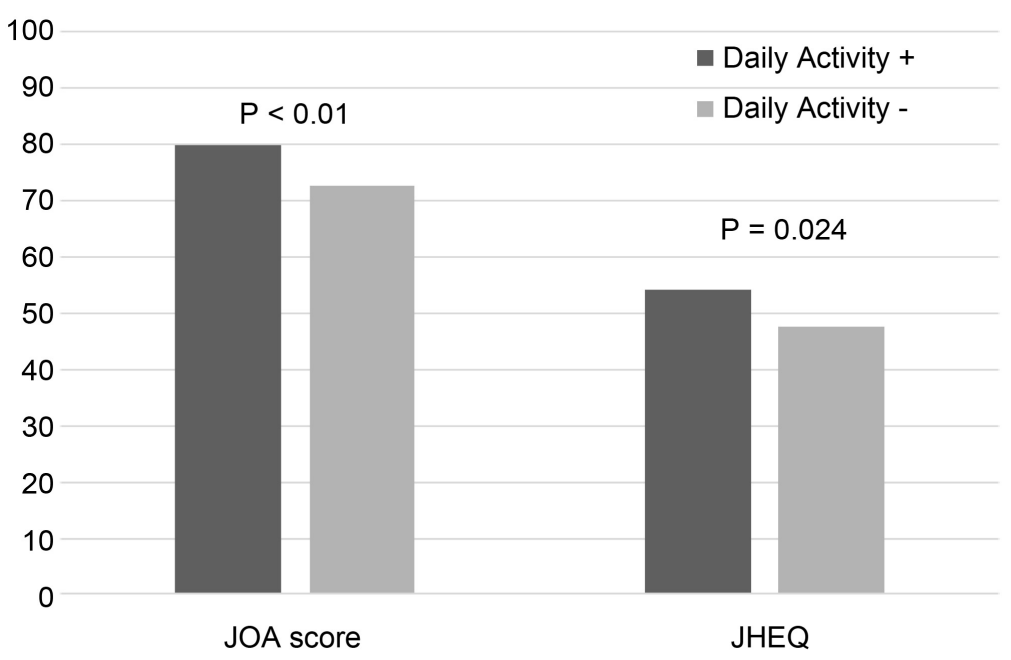

Mann-Whitney $U$ test

Figure 1. Relationships between daily activity and clinical results. Both JHEQ and JOA scores were significantly higher in the group resuming daily activities. 


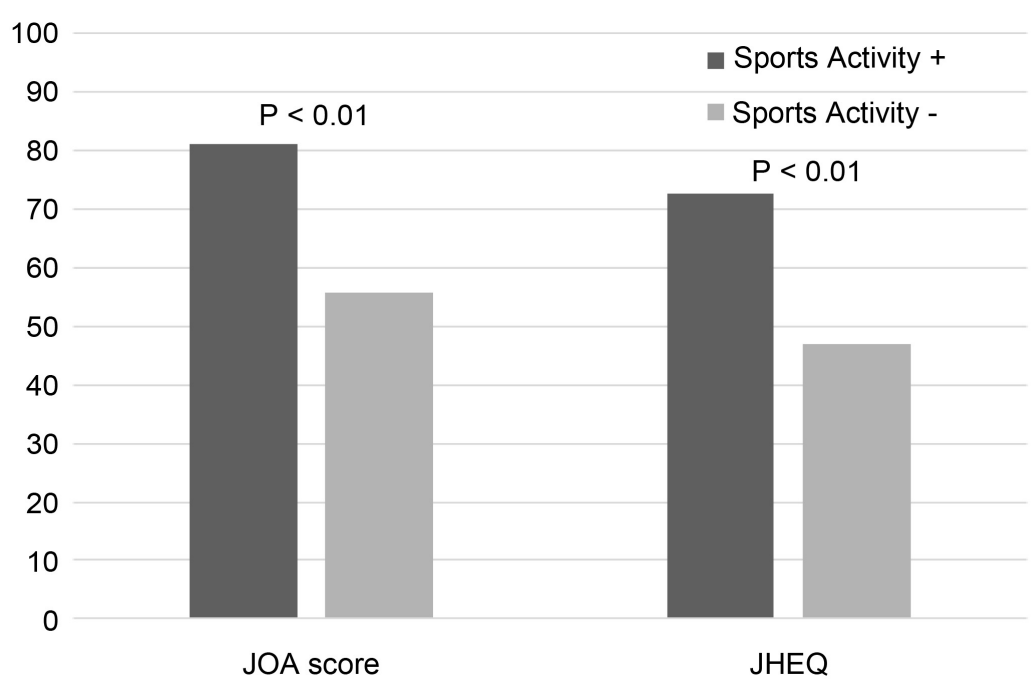

Mann-Whitney $U$ test

Figure 2. Relationships between sports activity and clinical results. Both JHEQ and JOA scores were significantly higher in the group resuming daily activities.

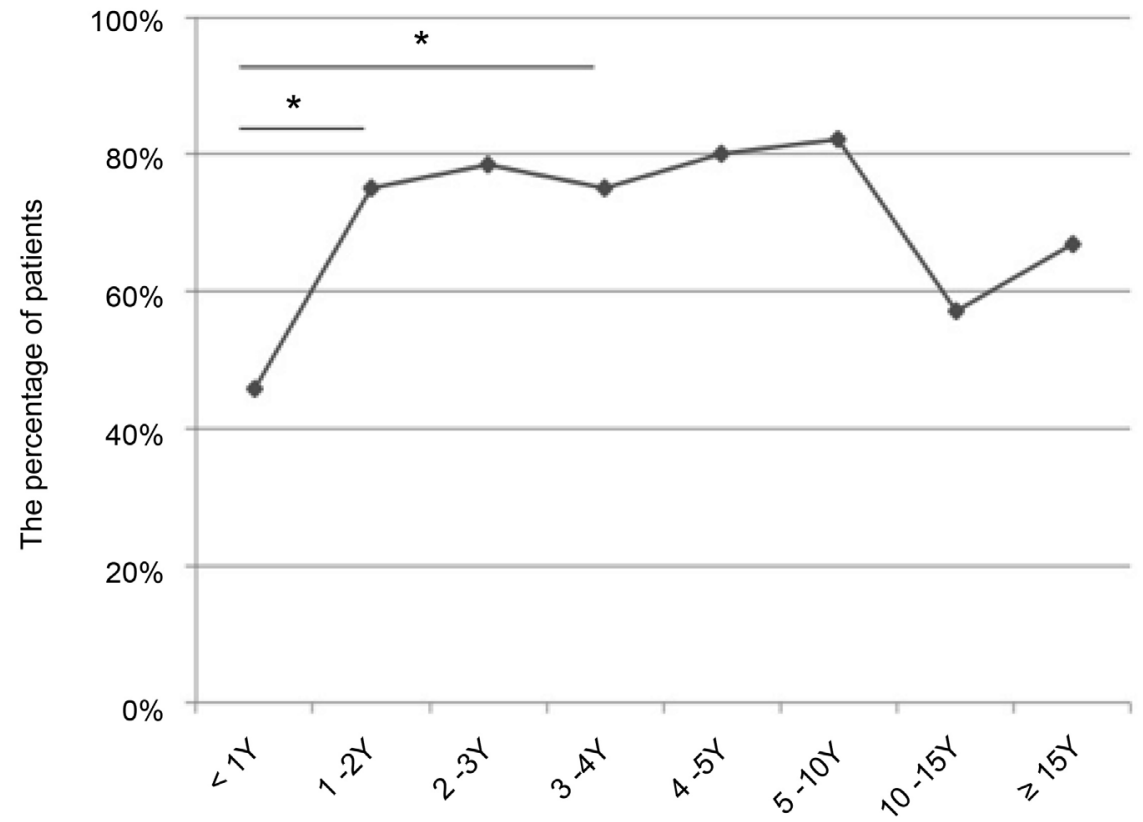

Post operative years

Figure 3. Relationship between the percentage of patients who did daily activities and the postoperative period $\left({ }^{*} \mathrm{P}<0.05\right)$.

Moreover, the relationship between the rate of patients who resumed sports activities and the postoperative period was examined. The rate increased after 1 year postoperatively. However, patients in the postoperative period $>5$ years but $<10$ years only had a significantly higher rate than those in the postoperative period $<1$ year. The patients in the postoperative period $>4$ years but $<5$ years were more involved in sports activities than those in the postoperative period $>3$ years 
but <4 years; however, there was no significant difference (Figure 4).

After THA, 35.8\% of patients had not resumed high-load daily activities. In particular, they gave up weeding from a primarily squatting posture $(25.8 \%)$ and snow shoveling with a snow scoop (18.2\%), since both these activities force hip joint flexion (Figure 5).

Furthermore, $19.9 \%$ of patients had not resumed sports activities after THA. In particular, they gave up cycling (11.0\%) and wild plant picking (6.5\%), which can also force hip joint flexion (Figure 6).

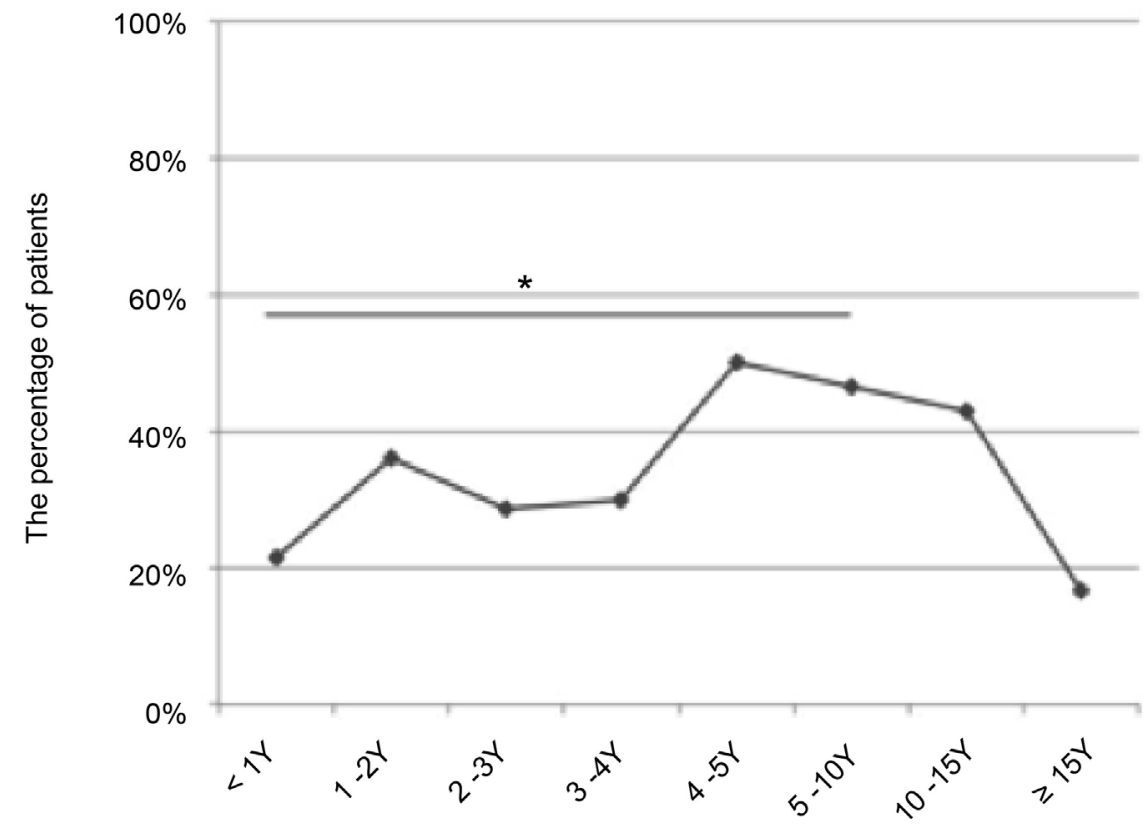

Post operative years

Figure 4. Relationship between the percentage of patients who did sports activities and the postoperative period $\left({ }^{*} \mathrm{P}<0.05\right)$.

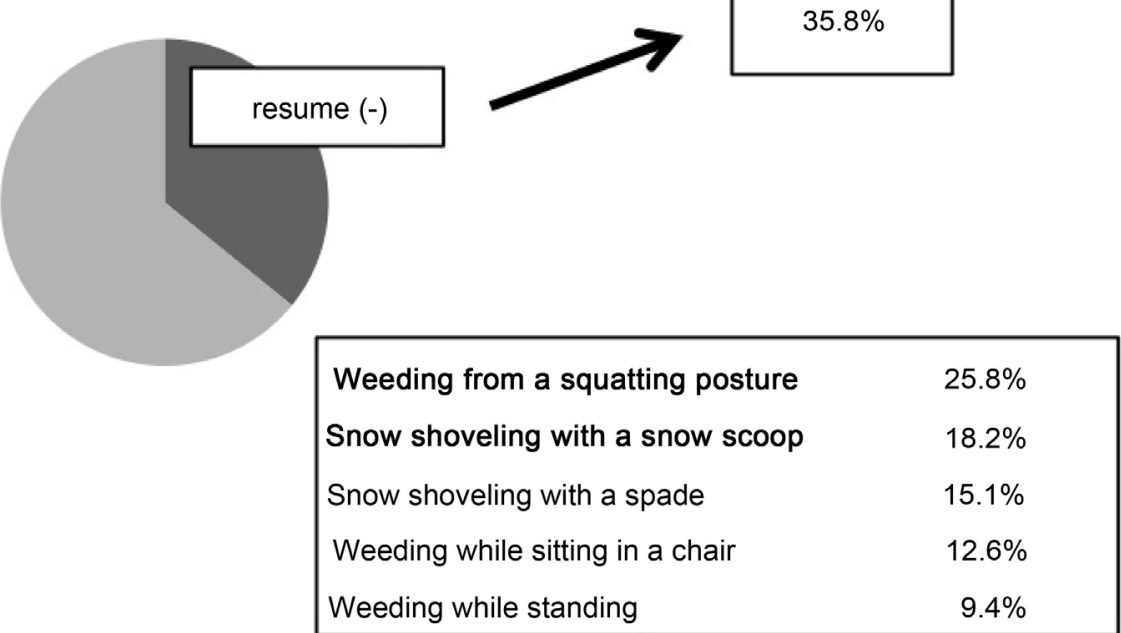

Figure 5. Rate and list of daily activities that patients did not resume. 


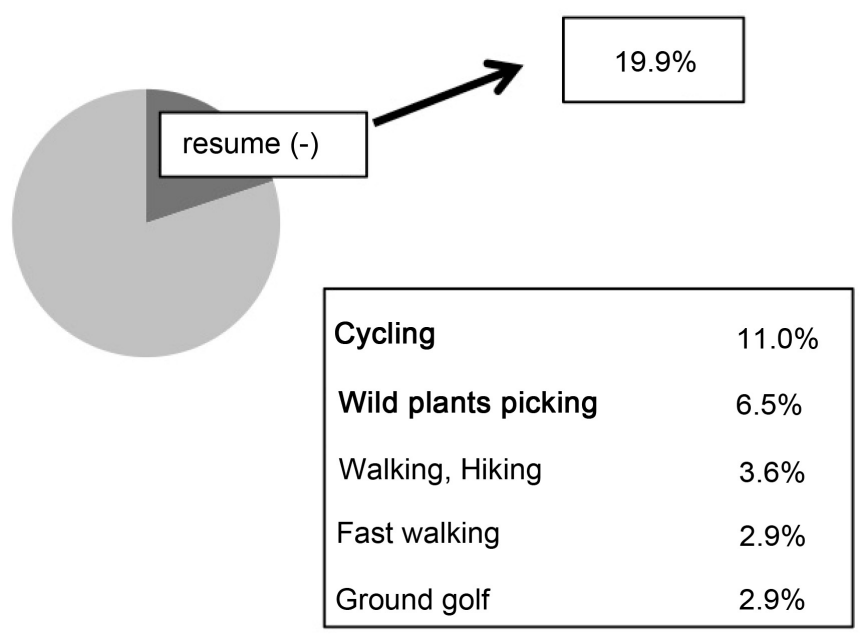

Figure 6. Rate and list of sports activities that patients did not resume.

The most common reason for giving up these activities was fear of implant failure (24.5\%), followed by concerns about dislocation (19.5\%). Other patients reported that they gave up these activities because of lack of permission from surgeons $(17.0 \%)$.

\section{Discussion}

To the best of our knowledge, this study is the first to evaluate the rates of resuming high-load daily activities and sports activities including weeding and snow shoveling after THA, along with an investigation of the reasons why patients could not resume them in farming areas and regions with heavy snowfalls.

The present study showed that about $70 \%$ of patients resumed high-load daily activities within 1 year of surgery, and about 50\% resumed sports activities. Furthermore, both JHEQ and JOA scores were higher in cases that resumed high-load daily activities including weeding and snow shoveling than in cases who did not resume them. This suggested that these activities did not increase the likelihood of periprosthetic fracture, dislocation, wear, or loosening of the prosthesis.

Kijima et al. previously investigated how many patients restarted daily activities such as weeding and snow shoveling and the relationship between the clinical results of total hip replacement and postoperative activities [8]. They reported that about $40 \%$ of patients resumed snow shoveling and weeding, and that their JOA scores were significantly higher than of those who did not resume these activities. In the present study, the number of patients was more than double compared to the above paper, and when the patients began high-load activities was also investigated. Furthermore, the reasons patients could not start high-load activities were examined. Thus, the present study provides very useful findings concerning education for patients receiving THA.

On the other hand, in 2012, Uchida studied 408 patients more than 1 year af- 
ter THA. Over half of the patients, including older individuals, played sports regularly. However, there were no early complications, such as fracture, dislocation, or migration of the cup or stem [9]. In 2017, Czech et al. reported that hip joint arthroplasty in people with degenerative joint diseases had a beneficial effect on their level of functioning and physical activity [10]. In addition, Ortmaier et al. reported that about $80 \%$ of patients returned to sports after short-stem THA, with almost no pain in the affected hip [11].

However, in the present study, about half of the patients gave up activities because of concern about dislocation or damage of their prosthesis, not because of pain or limitation of motion. In the future, it will be very important to explain to the patients that they do not necessarily need to limit daily and/or sports activities, which can improve overall satisfaction post-THA.

There are four main limitations in this study. First, this was an observational study based on a questionnaire survey and physical findings, which did not include imaging assessments. Second, because this was a multicenter study, a wide variety of surgical approaches and prosthesis models was used. Third, the analysis did not account for preoperative physical activity. To validate these results, prospective studies including standard surgical approaches, prosthesis models, and postoperative radiographs are needed. If we could conduct a long-term investigation of such a cohort, we would clarify the impact of sports and high-load activities on the implant. Fourth, this study did not evaluate range of motion, hip joint muscular strength, leg length difference, age, etc. A more extensive analysis is needed in the future, but since detailed data is lacking in this research, a prospective cohort study is necessary. In the future, by investigating these items and performing a multivariate analysis, it will become possible to investigate what kinds of patients can maintain high activity after the operation. This research provides evidence as the first step in obtaining such new findings.

\section{Conclusion}

About $70 \%$ of patients had resumed high-load daily activities after 1 year postoperatively. Furthermore, about half of the patients had resumed sports activities. Their clinical outcomes were better than of those who did not resume daily and sports activities. However, some patients gave up daily and sports activities because of concern about dislocation or damage of their hip prosthesis. Thus, if the patients were to be informed that there is no problem restarting high-load activities after THA, the patients could resume such activities, and their clinical outcomes may be improved.

\section{Declaration of Interest Statement}

The authors report no conflicts of interest.

\section{Conflicts of Interest}

The authors declare no conflicts of interest regarding the publication of this paper. 


\section{References}

[1] Meria, E.P. and Zeni, J.Jr. (2010) Sports Participation Following Total Hip Arthroplasty. International Journal of Sports Physical Therapy, 9, 839-850.

[2] Mariconda, M., Galasso, O., Costa, G.G., Recano, P. and Cerbasi, S. (2011) Quality of Life and Functionality after Total Hip Arthroplasty: A Long-Term Follow-Up Study. BMC Musculoskeletal Disorders, 12, 222. https://doi.org/10.1186/1471-2474-12-222

[3] Lee, I.M., Shiroma, E.J., Lobelo, F., Puska, P., Blair, S.N. and Katzmarzyk, P.T. (20112) Effects of Physical Inactivity on Major Non-Communicable Disease Worldwide: An Analysis of Burden of Disease and Life Expectancy. Lancet, 380, 219-229. https://doi.org/10.1016/S0140-6736(12)61031-9

[4] Ravi, B., Croxford, R., Austin, P.C., Lipscombe, L., Bierman, A.S., Harvey, P.J. and Hawker, G.A. (2014) The Relation between Total Joint Arthroplasty and Risk for Serious Cardiovascular Events in Patients with Moderate-Severe Osteoarthritis: Propensity Score Matched Landmark Analysis. British Journal of Sports Medicine, 48, 1580. https://doi.org/10.1136/bjsports-2014-f6187rep

[5] Matsumoto, T., Kaneuji, A., Hiejima, Y., Sugiyama, H., Akiyama, H., Atsumi, T., Ishii, M., Izumi, K., Ichiseki, T., Ito, H., Okawa, T., Ohzono, K., Otuska, H., Kishida, S., Kobayashi, S., Sawaguchi, T., Sugano, N., Nakajima, I., Nakamura, S., Hasegawa, Y., Fukuda, K., Fujii, G., Mawatari, T., Mori, S., Yasunaga, Y. and Yamaguchi, M. (2012) Japanese Orthopaedic Association Diseases Evaluation Questionnaire (JHEQ): A Patient-Based Evaluation Tool for Hip-Joint Disease. The Subcommittee on Hip Disease Evaluation of the Clinical Outcome Committee of Japanese Orthopaedic Association. Journal of Orthopaedic Science, 17, 25-38. https://doi.org/10.1007/s00776-011-0166-8

[6] Healy, W.L., Iorio, R. and Lemons, M.J. (2001) Athletic Activity after Joint Replacement. The American Journal of Sports Medicine, 29, 377-388. https://doi.org/10.1177/03635465010290032301

[7] Murata, K. and Yano, E. (2002) Medical Statistics for Evidence-Based Medicine with SPBS User's Guide. Nankodo Publisher, Tokyo.

[8] Kijima, H., Yamada, S., Konishi, N., Kubota, H., Tazawa, H., Tani, T., Suzuki, N., Kamo, K., Okudera, Y., Sasaki, K., Kawano, T., Miyakoshi, N. and Shimada, Y. (2018) Relationship between Clinical Results of Total Hip Replacement and Postoperative Activities including Weeding and Snow Shoveling. Hip International, 28, 145-147. https://doi.org/10.5301/hipint.5000537

[9] Uchida, T. (2012) Investigation of Sports Activities for Patients of Postoperative Total Hip Arthroplasty with Osteoarthritis of the Hip. Kansetsugeka, 31, 58-65.

[10] Czech, S., Hermanson, J., Rodak, P., Stołtny, T., Rodak, Ł., Kasperczyk, S., Koczy, B. and Mielnik, M. (2017) Sports Activity Following Cementless Metaphyseal Hip Joint Arthroplasty. Journal of Human Kinetics, 60, 225-232. https://doi.org/10.1515/hukin-2017-0112

[11] Ortmaier, R., Pichler, H., Hitzl, W., Emmanuel, K., Mattiassich, G., Plachel, F. and Hochreiter, J. (2017) Return to Sport after Short-Stem Total Hip Arthoplasty. Clinical Journal of Sport Medicine, Epub Ahead of Print. https://doi.org/10.1097/JSM.0000000000000532 